\title{
Surgical wound monitoring by MRI with a metamaterial-based implanted local coil
}

\author{
Hanan Kamel $^{1}$, Richard R.A. Syms ${ }^{1, *}$, Evdokia M. Kardoulaki ${ }^{1}$, and Marc Rea ${ }^{2}$ \\ ${ }^{1}$ Imperial College London, EEE Department, Exhibition Road, SW7 2AZ London, UK \\ ${ }^{2}$ Imperial College Healthcare NHS Trust, Dept. of Radiology, Praed St., Paddington, W2 1NY London, UK
}

Received: 26 August 2017 / Accepted: 18 December 2017

\begin{abstract}
An implantable sensor for monitoring surgical wounds after bowel reconstruction is proposed. The sensor consists of a coupled pair of 8-element magneto-inductive ring resonators, designed for mounting on a biofragmentable anastomosis ring to give a local increase in signal-to-noise ratio near an annular wound during ${ }^{1} \mathrm{H}$ magnetic resonance imaging. Operation on an anti-symmetric spatial mode is used to avoid coupling to the $B_{1}$ field during excitation, and a single wired connection is used for MRI signal output. The electrical response and field-of-view are estimated theoretically. Prototypes are constructed from flexible elements designed for operation at $1.5 \mathrm{~T}$, electrical responses are characterized and local SNR enhancement is confirmed using agar gel phantoms.
\end{abstract}

Keywords: Metamaterial / MRI / local coil

\section{Introduction}

Anastomosis is a surgical procedure to restore luminal continuity after bowel tumour resection. A variety of techniques are used, including suturing, stapling, tissue fusion and compression rings [1]. Despite continuing advances, leakage remains a potentially devastating complication, with a high (6-22\%) mortality following surgery [2]. The cause is poor blood supply (ischemia), aggravated by mechanical tension and tissue damage from radiation therapy [3]. Leakage can lead to extended hospitalisation, multiple morbidities and poor functional outcome [4]. Strenuous efforts have been made to identify methods of leak detection [5]. Systemic indicators include pain tympanic abdomen, temperature, tachycardia, hypotension and low urine output. More specific indicators include intramucosal $\mathrm{pH}$ [6], intraperitoneal cytokines [7] and elevated serum C-reactive protein [8]. However, these are unreliable. Generally, leaks are therefore only identified when they are fully developed, using computed tomography [9]. At this point, the prognosis is often poor.

The severity of the problem justifies a continued search for sensing modalities capable of detecting advance warning of conditions likely to lead to a leak, such as ischemia. Targeted intervention might then lead to greater retention of uncontaminated, viable tissue for the salvage

\footnotetext{
* e-mail: r.syms@imperial.ac.uk
}

operation, improving the potential outcome. One possibility is magnetic resonance spectroscopy (MRS). ${ }^{1} \mathrm{H}$ and ${ }^{31} \mathrm{P}$ MRS have been used to monitor the development of colonic ischemia in animal experiments, based on a transient increase in lactate [10] or a reduction in the $\alpha, \beta$ and $\gamma$ adenosine triphosphate peaks relative to phosphocreatine [11]. Unfortunately, due to the susceptibility of even the best external coils to body noise $[12,13]$, MRS has a low signal-to-noise ratio (SNR), which leads to poor results in the abdomen. However, SNR may be increased using internal coils with a low field-of-view (FOV) for body noise $[14,15]$.

The aim of this work is to investigate whether similar principles could be exploited in transient sensors designed to anticipate anastomotic leak following bowel reconstruction. The sensors are based on thin-film coils, adapted to the local anatomy, and designed to boost SNR during MRI or MRS carried out in hospital following surgery. The coil design is based on magneto-inductive (MI) waveguides $[16,17]$, exploiting their lack of connection between the resonant elements to allow fragmentability. A coupled pair of waveguides [18] and a ring resonant geometry $[19,20]$ are used to obtain decoupling and a FOV matched to annular wounds. The resulting sensor has the potential for eventual implantation in a two-part biofragmentable anastomosis ring (BAR) [21,22]. The electrical design is described in Section 2, and the FOV is discussed in Section 3. Bench testing and MRI evaluation of prototypes at $1.5 \mathrm{~T}$ are described in Section 4, and conclusions are drawn in Section 5 . 


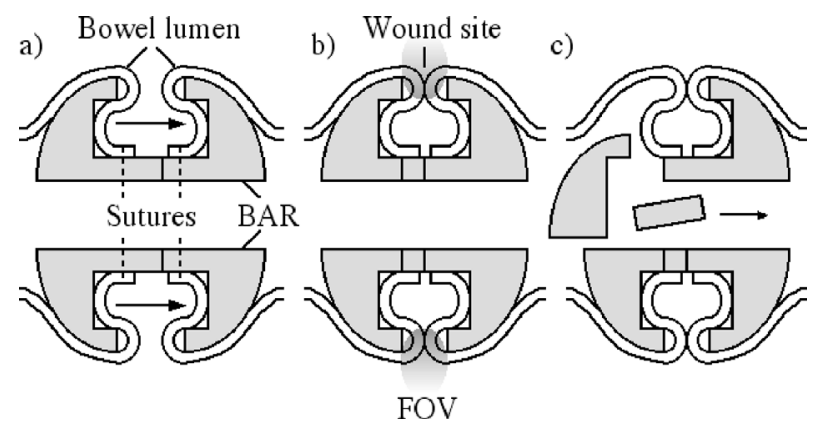

Fig. 1. (a) Implantation, (b) operation and (c) breakdown of a biofragmentable anastomosis ring.

\section{Electrical design}

In this section we present the initial electrical design of an implantable sensor for annular wound monitoring using locally enhanced magnetic resonance imaging.

\subsection{Biofragmentable anastomosis ring}

Figure 1 shows a BAR, which consists of two connecting rings of biodegradable polymer designed to act as a mechanical support for the sections of bowel to be joined, promoting healing by ensuring good perfusion. The sections are first attached to the two halves of the BAR with sutures (Fig. 1a). The BAR is then assembled to hold the tissue surfaces together under gentle compression (Fig. 1b). After around two weeks, the BAR breaks down into fragments that are excreted naturally (Fig. 1c).

\subsection{Implantable resonator}

The desired FOV of any implanted coil for local enhancement of MRI signals around the surgical wound is clearly annular. Figure 2 shows two candidate arrangements. One possibility is a MI ring, a resonant structure based on a section of waveguide formed into a polygon as shown in Figure 2a. The ring can couple to the rotating field of precessing nuclear dipoles and has a cylindrical FOV. However, it can also couple to the $B_{1}$ field during the excitation phase of MRI, potentially giving rise to overexcitation artefacts. To avoid this, a coupled pair of rings operating on their lowest-order anti-symmetric spatial mode (which cannot couple to spatially uniform RF magnetic fields) may be used as shown in Figure 2b, with each ring embedded in one half of a BAR.

Ideally, the individual elements would be biodegradable. Conducting polymers based on polypyrrole nanoparticles or carbon nanotubes in a biodegradable matrix have been developed [23] and are being used in wireless implantable sensors [24]. However, their electrical performance is low, and their toxicity uncertain [25]. We therefore propose the use of conventional materials to form the resonant elements. Due to the lack of physical connections between elements, the rings can still separate during biofragmentation.

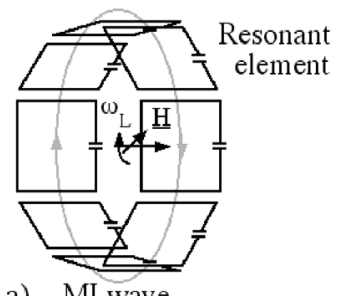

a) $\mathrm{MI}$ wave

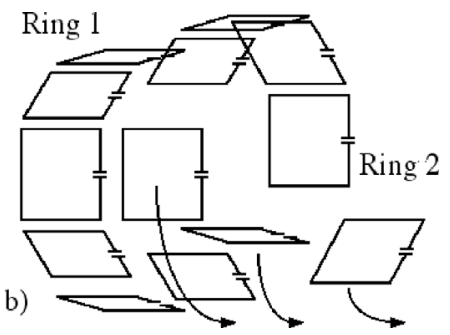

Fig. 2. (a) Single and (b) double magneto-inductive ring resonators.

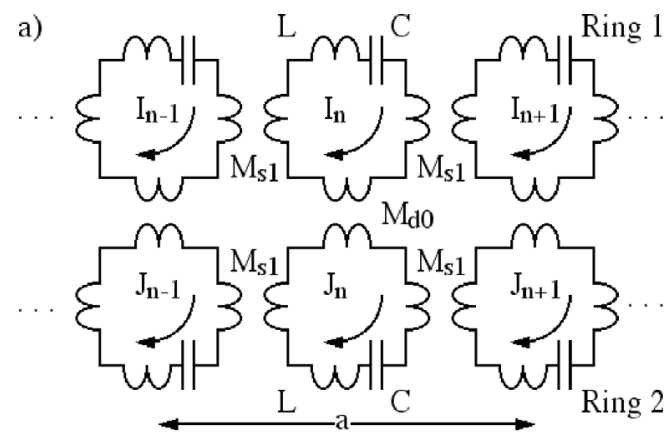

b) Intra-ring coefficients

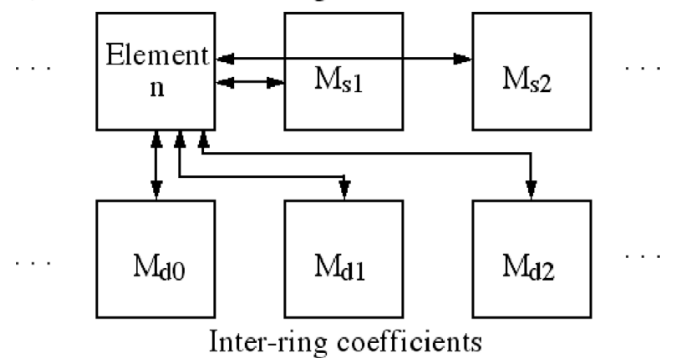

Fig. 3. (a) Equivalent circuit of lossless double-ring system, with nearest-neighbour coupling only; (b) definition of non-nearestneighbour coupling coefficients.

\subsection{Equivalent circuit and dispersion relation}

Figure 3a shows the equivalent circuit for a lossless double MI ring system with nearest neighbour coupling. Each ring is formed from a set of $N$ elements with self-inductance $L$ and capacitance $C$, with intra-ring and inter-ring mutual inductances $M_{\mathrm{s} 1}$ and $M_{\mathrm{d} 0}$. Assuming loop currents $I_{n}$ and $J_{n}$ in the $n$th elements of rings 1 and 2 , and that the circuits are infinitely long, the circuit equations at angular frequency $\omega$ in the absence of voltages are [18]:

$$
\begin{aligned}
& (j \omega L+1 / j \omega C) I_{n}+j \omega M_{s 1}\left(I_{n-1}+I_{n+1}\right)+j \omega M_{d 0} J_{n}=0 \\
& (j \omega L+1 / j \omega C) J_{n}+j \omega M_{s 1}\left(J_{n-1}+J_{n+1}\right)+j \omega M_{d 0} I_{n}=0 .
\end{aligned}
$$

Assumption of travelling wave solutions in the form $I_{n}=I_{0} \exp (-\mathrm{j} n k a)$ and $J_{n}=J_{0} \exp (-\mathrm{j} n k a)$, where $k$ is the propagation constant and $a$ is the element spacing, then yields:

$$
\begin{aligned}
& \left\{1-\omega_{0}^{2} / \omega^{2}+\kappa_{s 1} \cos (k a)\right\} I_{0}+\left(\kappa_{d 0} / 2\right) J_{0}=0 \\
& \left\{1-\omega_{0}^{2} / \omega^{2}+\kappa_{s 1} \cos (k a)\right\} J_{0}+\left(\kappa_{d 0} / 2\right) I_{0}=0 .
\end{aligned}
$$


Here $\omega_{0}=1 / \sqrt{ }(L C)$ is the angular resonant frequency of the loops, and $\kappa_{s 1}=2 M_{s 1} / L$ and $\kappa_{d 0}=2 M_{\mathrm{d} 0} / L$ are intraring and inter-ring coupling coefficients. Equation (2) can be decoupled to yield a dispersion equation that is quadratic in $\omega^{2}$. Consequently, the dispersion diagram must have two branches. However, overall symmetry implies that the current patterns must be exactly symmetric $\left(J_{0}=I_{0}\right)$ and anti-symmetric $\left(J_{0}=-I_{0}\right)$, allowing the dispersion equations for the two branches to be extracted separately, as:

$$
\omega / \omega_{0}=1 /\left\{1+\kappa_{s 1} \cos (k a) \pm \kappa_{d 0} / 2\right\} .
$$

More generally, there may be mutual inductance between non-nearest neighbours in the same ring and in different rings. In this case, it is simple to show that the dispersion equations for the two branches modify to:

$\omega / \omega_{0}=$

$1 / \sqrt{\left\{1+{ }_{m} \sum \kappa_{\mathrm{sm}} \cos (m k a) \pm\left[\kappa_{d 0} / 2+{ }_{m} \sum \kappa_{\mathrm{dm}} \cos (m k a)\right]\right\}}$.

Here $\kappa_{\mathrm{sm}}=2 M_{\mathrm{sm}} / L, \kappa_{\mathrm{dm}}=2 M_{\mathrm{dm}} / L, M_{\mathrm{sm}}$ and $M_{\mathrm{dm}}$ are the mutual inductances shown in Figure $3 \mathrm{~b}$, and the summation is taken over the number of interactions assumed. However, useful initial conclusions may be reached from a nearest-neighbour model.

\subsection{Decoupling and MRI signal detection}

For example, Figure 4 shows the dispersion diagram for typical planar coupling coefficients of $\kappa_{s 1}=-0.2$ and $\kappa_{d 0}=-0.1$. In this case, backward waves propagate in both rings. For ring resonance, the propagation constant must satisfy $k a=2 \pi \mu / N$ where $\mu=0,1 \ldots$ is the mode number [19]. For $N=8$ (for example) there are ten modes overall, five symmetric and five anti-symmetric, at $k a=0$, $\pi / 4, \pi / 2,3 \pi / 4$ and $\pi$. If the system is tuned to place the lowest-order ( $\mu=1$, arrowed) anti-symmetric resonance at the Larmor frequency $\omega_{L}$, this mode will not be excited by uniform $B_{1}$ fields and will remain decoupled during the excitation phase of MRI. However, it can still respond to the non-uniform fields generated during the detection phase, and the MRI signal can be extracted using taps connected to one or more elements.

Unfortunately, the second order symmetric resonance ( $\mu=2$, circled) has almost exactly the same frequency. Consequently, it may easily couple to the $B_{1}$ field. However, improved decoupling can be obtained if the intra-ring coupling $\kappa_{s 1}$ is made positive. In this case, forward waves are obtained. As a result, the dispersion curves slope upwards, with improved separation of the primary anti-symmetric resonance from nearby modes.

Rather than show this through the dispersion diagram, we present a circuit simulation. We assume the arrangement of Figure 5a. Here only one ring is shown, with the second being implied. All elements contain additional resistors $R$. Each ring is coupled by mutual inductance $M_{t}$

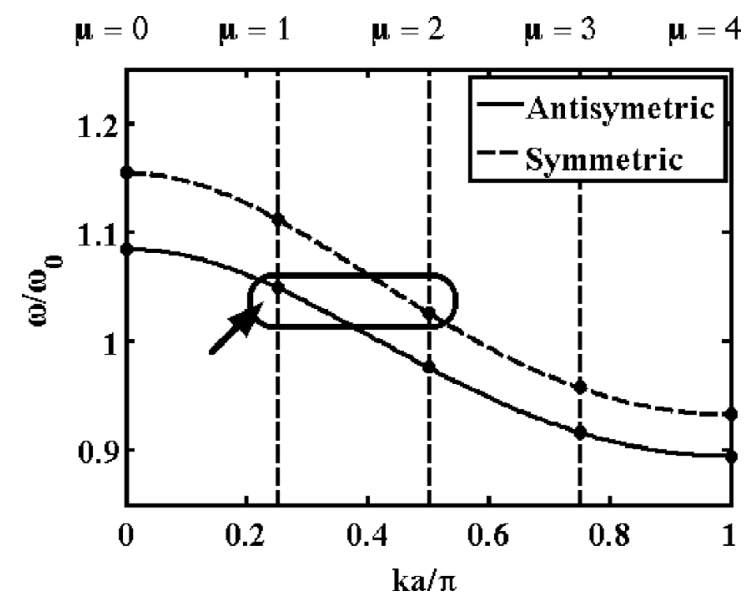

Fig. 4. Dispersion diagram for double-ring system with negative $\kappa_{s 1}$ and negative $\kappa_{d 0}$.

to a pair of loop probes of inductance $L_{t}$ on either side of the diameter, with the first containing a source with $50 \Omega$ output impedance and the second a $50 \Omega$ load. This arrangement mimics testing with an electronic network analyser.

The probes have two configurations. The first has equal coupling to each ring, and hence is designed only to excite and detect symmetric modes. The second has equal and opposite coupling to each loop, and hence will only interact with anti-symmetric modes. In each case, the circuit equations are simple to derive, and can be written in the form $V=\mathrm{Z} I$, where $\mathrm{Z}$ is an $18 \times 18$ matrix of impedances and $\bar{V}$ and $I$ are $\overline{1}$-element vectors of voltages and currents. The currents may be found by matrix inversion, assuming a voltage source in the input transducer, and the scattering parameters $S_{11}$ and $S_{21}$ may then be found by standard methods.

Figure 5b shows the frequency dependence of transmission thus obtained, assuming nearest-neighbour coupling coefficients $\kappa_{s 1}=+0.2$ and $\kappa_{d 0}=-0.1$, a quality factor $Q=\omega_{0} L / R=100$, and arbitrary weak coupling to the probes. For each probe configuration, five resonant peaks may be seen, and the resonant frequency of the elements has been adjusted to place the $\mu=1$ anti-symmetric mode at $63.85 \mathrm{MHz}$, the Larmor frequency for ${ }^{1} \mathrm{H} \mathrm{MRI}$ at $1.5 \mathrm{~T}$. The two responses are similar, but the symmetric mode spectrum is up-shifted in frequency. As a result, symmetric mode excitation is reduced by around $25 \mathrm{~dB}$ at the Larmor frequency.

To achieve a positive intra-ring mutual inductance, adjacent elements are merely required to overlap in a quasiaxial configuration. Figure 6a shows a suitable inductor track layout, unfolded. Here inductors $L$ are patterned on either side of a flexible substrate, with additional capacitors $C$ added as surface mount components. Inductors from the same ring overlap, while those from different rings lie side by side. For comparison with later experiments we assume the output is directly coupled to the scanner input (a load $Z_{0}$ ) from a single element via matching and tuning capacitors $C_{M}$ and $C_{T}$. However, wireless transmission of the signal will ultimately be required. Figure $6 \mathrm{~b}$ shows the circuit as a ring on a cylindrical mount. 


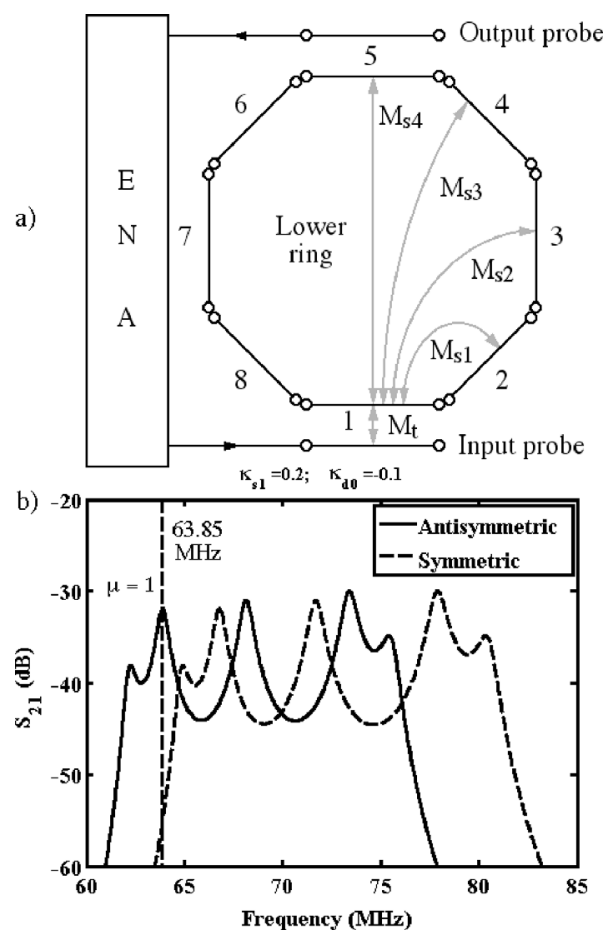

Fig. 5. (a) Arrangement for circuit simulation (only single ring shown); (b) frequency dependence of $S_{21}$ for double-ring system with positive $\kappa_{s 1}$ and negative $\kappa_{d 0}$.

\section{$3 \mathrm{MRI}$ signal reception pattern}

In this section, we estimate the reception pattern, which can be estimated from reciprocity by considering the magnetic field generated with unit current at the coil terminals [12].

\subsection{Signal reception in MRI}

We assume the sensor has its axis parallel to the $z$-axis, the direction of the static field $B_{0}$. In the transverse plane, the coil field can be written as $\underline{B}_{\mathrm{xy}}=\mu_{0}\left(H_{x} \underline{u}_{\mathrm{x}}+H_{y} \underline{u}_{\mathrm{y}}\right)$, where $\mu_{0}$ is the free space permeability and $\bar{u}_{\mathrm{x}}$ and $\underline{u}_{\mathrm{y}}$ are unit vectors in the $x$ - and $y$-directions. The complex signal voltage $V_{S}$ induced in the coil by a volume $d v$ of dipoles is then $V_{S}=-j \omega_{L} \underline{B}_{\mathrm{xy}} \cdot \underline{M}_{\mathrm{xy}} d v$. Here $\underline{M}_{\mathrm{xy}}=M\left(\underline{u}_{\mathrm{x}}+j \underline{u}_{y}\right)$ is the transverse magnetization and $M=M_{0}$ for a $90^{\circ}$ flip angle, where $M_{0}$ is the equilibrium magnetization per unit volume. When $H$ is real, is simple to show that:

$$
\left|V_{S}\right|=\omega_{L} \mu_{0} M_{0} \sqrt{\left(H_{x}^{2}+H_{y}^{2}\right)} d v
$$

Consequently, the spatial variation of the function $\sqrt{ }\left(H_{x}^{2}+H_{y}^{2}\right)$ defines the reception pattern.

\subsection{Field-of-view}

For accurate results a 3D simulation of the exact geometry is required. Here, we use simpler 2D approximations for a single ring. We assume that the elements are located on a cylindrical scaffold of radius $r_{0}$, and that each element can
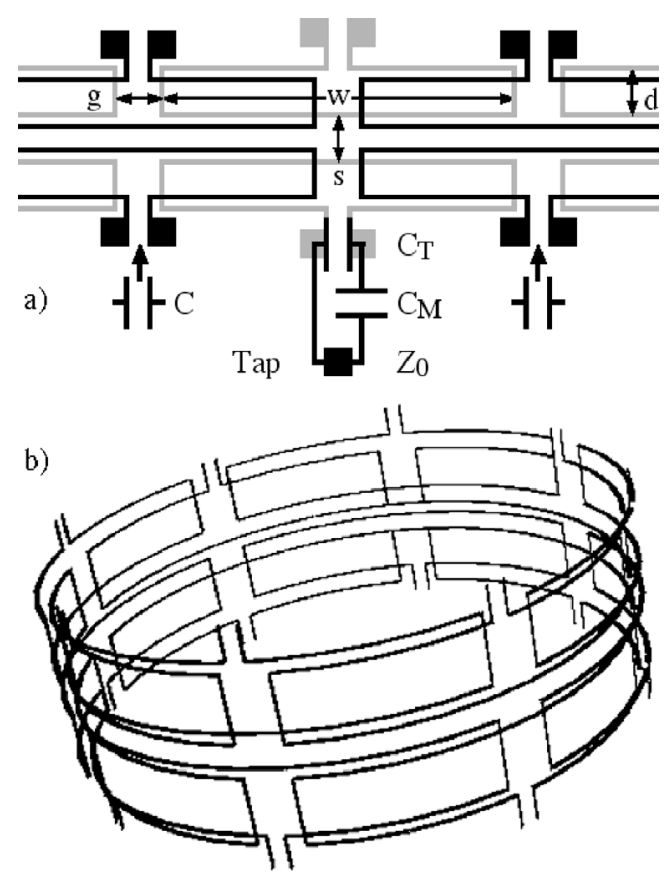

Fig. 6. PCB layout of prototype sensor, a) flat, and b) mounted on cylindrical scaffold.

be modeled as a pair of cylindrical wires passing through the points $\left(x_{n 1}, y_{n 1}\right)$ and $\left(x_{n 2}, y_{n 2}\right)$, given by:

$$
\begin{aligned}
& x_{n 1,2}=r_{0} \cos \left(\theta_{n} \pm \Delta \theta\right) \\
& y_{n 1,2}=r_{0} \sin \left(\theta_{n} \pm \Delta \theta\right) .
\end{aligned}
$$

Here $\theta_{n}=2 \pi(n-1) / N-\pi / 2$ (so element 1 is at the base of the coil) and $\Delta \theta=f \pi / N$ (so the coefficient $f$ defines the degree to which the resonant elements fill the ring circumference). A filling factor $f \approx 1$ corresponds to the planar geometry, while $f \approx 2$ corresponds to the overlapping arrangement needed for improved decoupling. For a single tap at $n=1$, we assume the conductors carry currents $\pm I_{n}$, where $I_{n}$ is the standing wave pattern:

$$
I_{n}=-\sin \left(\theta_{n}\right) .
$$

Equation (7) implies that the coil currents vary, and that two of them $\left(I_{3}\right.$ and $\left.I_{7}\right)$ are zero when $N=8$. Consequently, we would expect a reduction in the N-fold symmetry of the reception pattern. The $x$ - and $y$-components $H_{x}$ and $H_{y}$ of the magnetic field are then given by:

$$
\begin{aligned}
H_{x}= & { }_{n=1} \sum^{N}\left(I_{n} / 2 \pi\right)\left[\left(y-y_{n 1}\right) /\left\{\left(x-x_{n 1}\right)^{2}+\left(y-y_{n 1}\right)^{2}\right\}\right. \\
& \left.-\left(y-y_{n 2}\right) /\left\{\left(x-x_{n 2}\right)^{2}+\left(y-y_{n 2}\right)^{2}\right\}\right] \\
H_{y}= & n=1 \sum^{N}\left(I_{n} / 2 \pi\right)\left[\left(x-x_{n 1}\right) /\left\{\left(x-x_{n 1}\right)^{2}+\left(y-y_{n 1}\right)^{2}\right\}\right. \\
& \left.-\left(x-x_{n 2}\right) /\left\{\left(x-x_{n 2}\right)^{2}+\left(y-y_{n 2}\right)^{2}\right\}\right] .
\end{aligned}
$$

Figure $7 \mathrm{a}$ shows a contour map of $\sqrt{ }\left(H_{x}^{2}+H_{y}^{2}\right)$ obtained for an 8-element ring with a filling factor of $f=1.8$. The coil structure is superimposed in red, with 

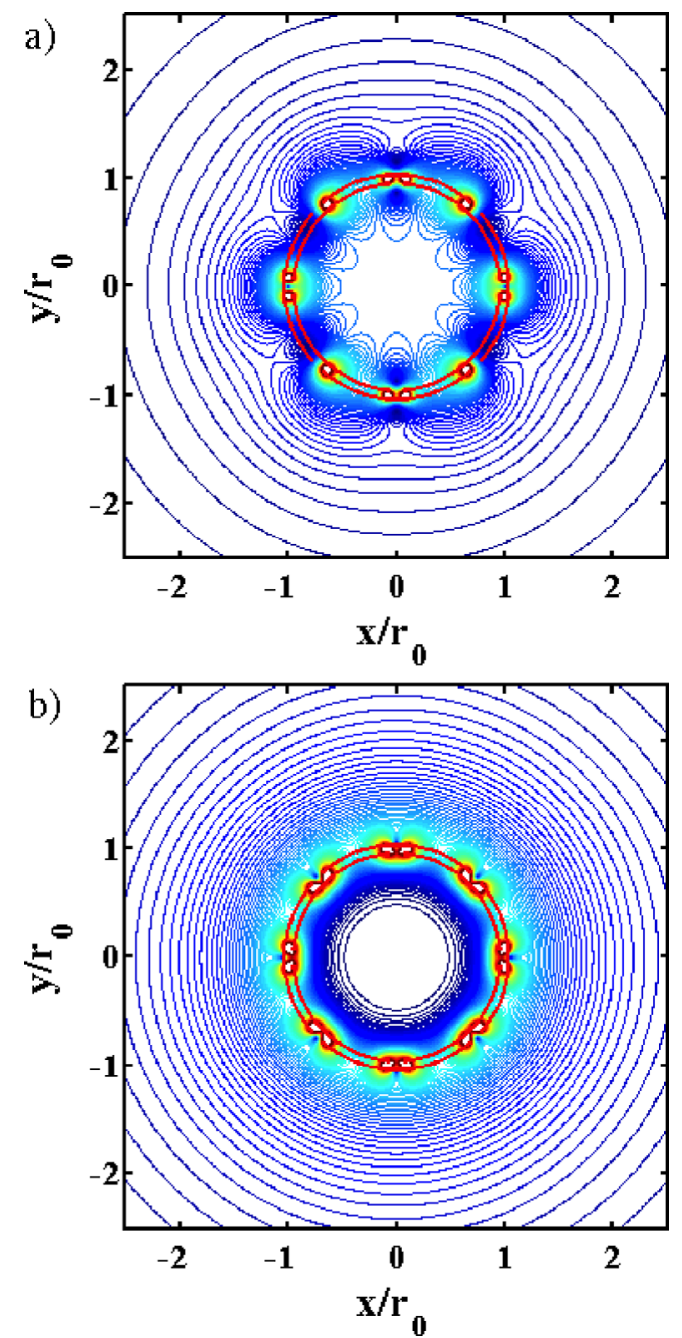

Fig. 7. 2D sensitivity profiles for a single MI ring with a filling factor $f=1.8$, with (a) single and (b) quadrature taps.

alternate elements radially offset for clarity. Inside the ring, the sensitivity is approximately uniform. Outside, there are sensitivity peaks near the coil conductors, but the angular variation reduces at large distance. Here the sensitivity falls off approximately as $1 / r^{2}$, and so must fall below that of an external coil with uniform sensitivity at some radius. The reduction in symmetry predicted above is also evident, but this again reduces with distance. Symmetry may be improved using quadrature taps. In this case we may take the current variation $I_{n}$ as a complex exponential instead of a sinusoid. Since the magnetic fields are now complex, sensitivity depends on the alternative function $\sqrt{ }\left\{\left(H_{x r}-H_{y i}\right)^{2}+\left(H_{y r}+H_{x i}\right)^{2}\right\}$. A similar calculation leads to the sensitivity pattern shown in Figure $7 \mathrm{~b}$, where 8-fold symmetry is clearly restored.

Of course, the reception pattern is further modified by the finite length of each coil, and by the fact that two rings are used, operating on an anti-symmetric mode. These additional features imply that the FOV will be finite in the axial direction, with a null between the two coils. Further work will clearly be required to establish the optimum design for wound sensing.

\section{Prototype construction and experimental evaluation}

In this section we describe construction of the prototype sensors and present the results of electrical characterisation and ${ }^{1} \mathrm{H}$ magnetic resonance imaging of phantoms at $1.5 \mathrm{~T}$.

\subsection{Construction}

Prototype sensors containing coupled 8-element MI rings with the track layout of Figure 6 a were constructed from lithographically patterned copper inductors and nonmagnetic surface mount capacitors on flexible plastic substrates. Each sensor was formed from two overlaid substrates containing four resonators from each ring, with the tracks from adjacent resonators laterally offset to reduce parasitic capacitance. The outer substrate was cut away to allow access to solder pads on the inner substrate.

The substrates were mounted on an annular nylon support with an oversize diameter $(50 \mathrm{~mm})$ and length $(30 \mathrm{~mm})$ to improve resonant performance and assist electrical diagnosis. Smaller rings were also investigated, but these had lower $Q$-factors and consequently less distinct mode spectra. The following inductor dimensions were used: a loop depth $d=6 \mathrm{~mm}$ and width $w=35 \mathrm{~mm}$, an intra-ring gap $g=4 \mathrm{~mm}$ (giving a filling factor $f=2 w$ / $(w+\mathrm{g}) \approx 1.8$ as previously assumed $)$, a track width of $0.5 \mathrm{~mm}$ and a track thickness of $0.035 \mathrm{~mm}$. The inter-ring separation s was approximately $3 \mathrm{~mm}$.

\subsection{Electrical characterisation}

The inductance was first extracted as $L=60 \mathrm{nH}$ by measuring the resonant frequencies of single elements with known capacitors, using a network analyser and inductive probes. Complete circuits were then populated with identical capacitors $C$, and resonant modes were identified by measurement of phase differences between elements around and between rings. The capacitor value was then adjusted to tune the $\mu=1$ anti-symmetric mode to $65 \mathrm{MHz}$ to allow for a reduction in resonant frequency to $63.85 \mathrm{MHz}$ on immersion in an agar gel phantom. Figure 8a shows a completed sensor at this point.

Transmission across the ring was then measured using inductive probes on either side of the diameter as previously shown in Figure 5a. Figure 8b shows the frequency variation of $S_{21}$ for symmetric and antisymmetric modes. As expected, only low-order antisymmetric modes have resonant frequencies near the Larmor frequency, suggesting that the design has indeed placed the symmetric modes out-of-band. The unloaded $Q$ factor of the $\mu=1$ anti-symmetric mode was measured as 80. A tap consisting of a short length of sub-miniature coaxial cable was then attached to one element, using capacitors $C_{T}$ and $C_{M}$ to match this mode to $50 \Omega$ impedance. Finally, the circuit was made waterproof using nail varnish. 

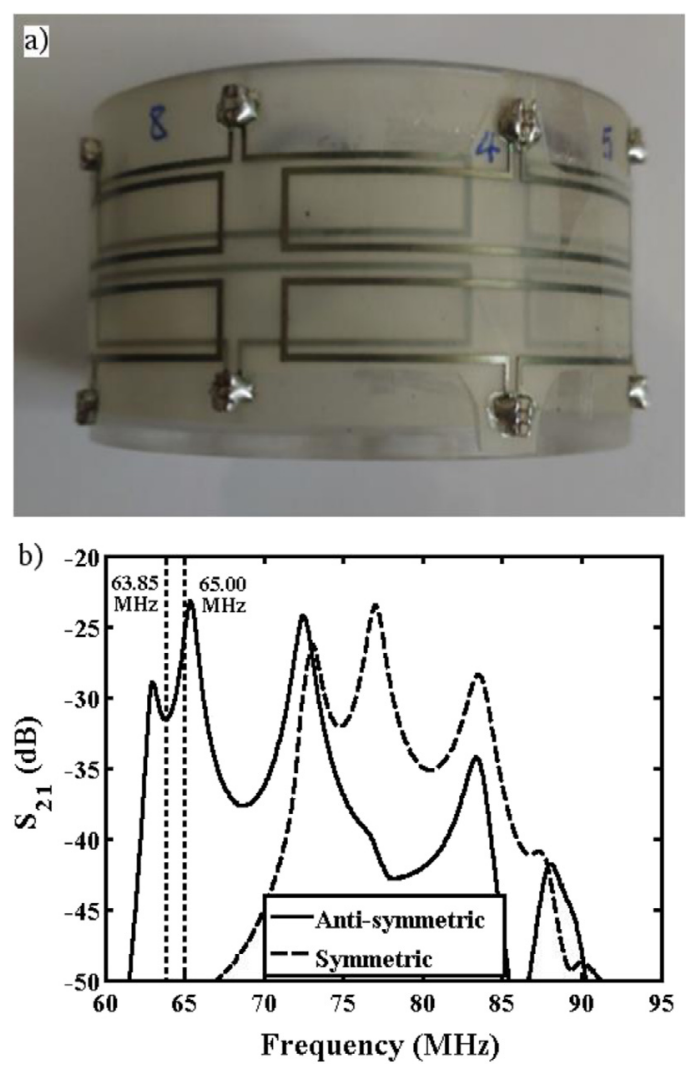

a)

Fig. 8. (a) Prototype sensor before encapsulation; (b) mode spectrum obtained by inductive probing.

\subsection{MR imaging}

Imaging was carried out with the sensor immersed in a gel phantom. The sensor was mounted on a support pillar as shown in Figure 9a, and placed inside a $70 \mathrm{~mm}$ diameter cylindrical mould as shown in Figure 9b. The mould was then filled with agar gel doped with $\mathrm{NiCl}_{2} \cdot 6 \mathrm{H}_{2} \mathrm{O}(3.37 \mathrm{~g} / \mathrm{L})$ and $\mathrm{NaCl}(2.4 \mathrm{~g} / \mathrm{L})$, giving tissue mimicking MR properties $\left(T_{1}=800 \mathrm{~ms}, T_{2}=50 \mathrm{~ms}\right)$. Imaging was carried out in a 1.5 T GE Signa Excite scanner, with the gel phantom lying horizontally on the patient bed between two cuboid phantoms as shown in Figure 9c, with the tap at its base. The system body coil was used for excitation and detection, and the sensor was connected to an auxiliary input for locally enhanced signal reception. A spin-echo sequence was used, with an excitation time $\mathrm{TE}=8.46 \mathrm{~ms}$ and a repetition time $\mathrm{TR}=520 \mathrm{~ms}$. Images were obtained as stacks of axial slices with a $2 \mathrm{~mm}$ thickness, a $0.2 \mathrm{~mm}$ separation, a $200 \mathrm{~mm}$ FOV, a $192 \times 160$ acquisition matrix and 8 excitations.

Figure 10a shows a slice image through one of the rings, obtained using the body coil. The two cuboid phantoms lie to the left and right, with the gel phantom between. Here, the grey disc defines the doped agar signal source, while the inner black annulus indicates the coil mount. Where there is signal, this image is of generally low but uniform brightness. There is some perturbation to the magnetization near the coil conductors, indicating that passive decoupling has been only partially successful. However, the range of this decoupling artefact is surprisingly short, considering the
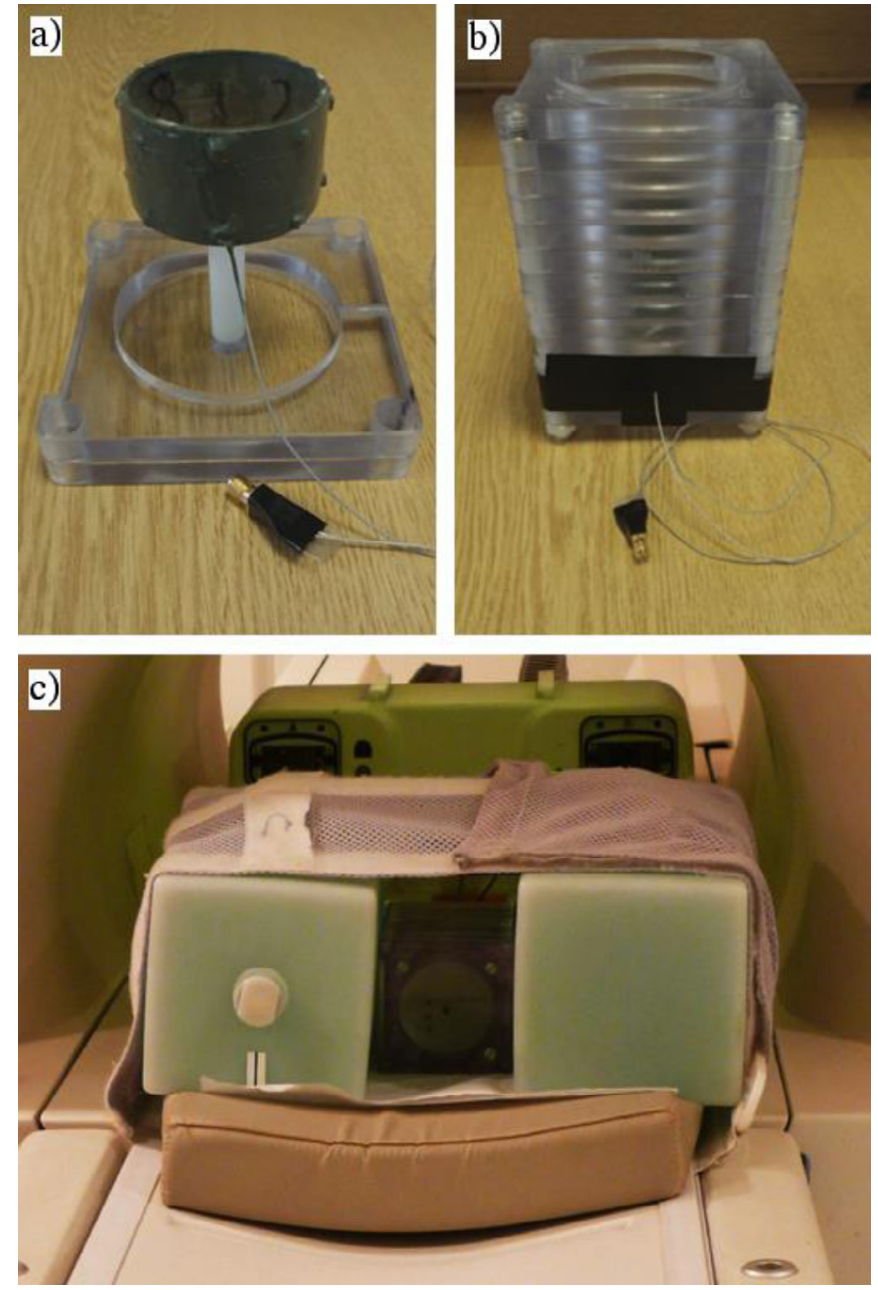

Fig. 9. Arrangement for (a) sensor mounting, (b) gel phantom moulding, and (c) magnetic resonance imaging.

intimate contact of the surrounding medium. The most likely explanation is lack of symmetry (either from elementto-element or from ring-to-ring, both of which will tend to mix the symmetric and anti-symmetric modes). This may arise from overlay errors, capacitor tolerance or the presence of a tap in one of the rings. Figure 10b shows the corresponding image obtained using the sensor. Only the gel phantom may now be seen, due to the limited radial FOV. However, within this region the image is significantly brighter - in fact, there is evidence of a minor clipping artefact running vertically. The lack of 8 -fold symmetry due to the use of a single tap may be seen.

SNR was found from the ratio of extracted signal to noise from a region of interest devoid of signal. Figure 10c compares the SNR of the body coil and the sensor along a vertical line through the centre of the gel phantom. The SNR of the former is around 40 at the centre of the phantom. The latter is approximately 3 times higher in the same region and over 15 times higher near the conductors, confirming the local signal enhancement. However, the over-excitation artefact previously mentioned has disrupted the sensitivity pattern outside the sensor, and further work is clearly required to minimise this effect. 

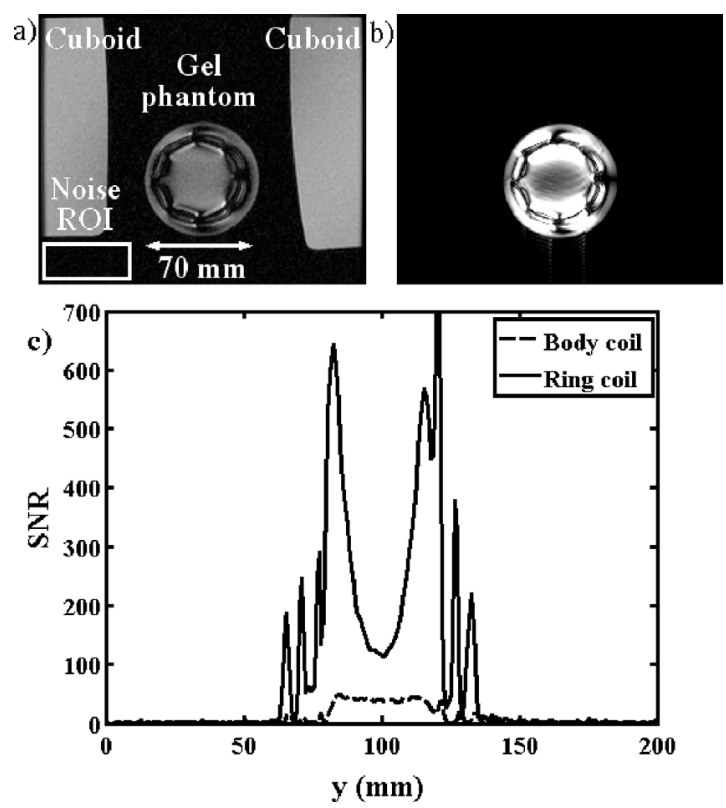

Fig. 10. Axial slice images obtained using (a) the body coil and (b) the sensor; (c) variation of SNR with vertical position obtained using the two coils.

\section{Conclusions}

A prototype internal sensor for surgical wound monitoring by MRI has been demonstrated, based on a coupled pair of thin-film MI ring resonators that are ultimately intended for incorporation in a BAR. The design has been chosen to maximise the frequency separation of resonant modes, with the sensor operating on the first anti-symmetric spatial mode to minimise coupling to the $B_{1}$ field during excitation. ${ }^{1} \mathrm{H}$ phantom imaging at $1.5 \mathrm{~T}$ shows moderately effective passive decoupling and the expected local signal enhancement. Although this represents an encouraging proof of concept, significant further work is required to demonstrate utility of the sensor in localised imaging or spectroscopy of annular wounds. Engineering development is required to improve decoupling, introduce quadrature taps, eliminate the wired connection used for signal output, optimise the FOV, investigate orientation effects, and develop a biofragmentable matrix. Testing is then required to confirm MRI safety and lack of toxicity. This work is in progress.

The Authors are extremely grateful to Mr Phil Jones for workshop support, and to Dr Rebecca Quest for additional MRI. Hanan Kamel is also grateful to Imperial College London for provision of a $\mathrm{PhD}$ studentship.

\section{References}

1. J.C. Slieker, I.M. Mulder, J. Jeekel, et al., JAMA Surg. 148, 191 (2013)

2. J. Pickleman, W. Watson, Cunningham, J. Am. Coll. Surg. 188, 473-482 (1999)

3. A. Karliczek, D.A. Benaron, C.J. Zeebregts, et al., J. Surg. Res. 152, 288 (2009)

4. R. Essani, R. Bergamaschi, Gastroenterologia Polska 16, 123 (2009)

5. K. Trencheva, K.P. Morrisey, M. Wells, et al., Ann. Surg. 257, 108 (2013)

6. M. Millan, E. Garcia-Granero, B. Flor, et al., Dis. Colon Rectum 49, 595 (2006)

7. B. Ugras, M. Giris, Y. Erbil, et al., Int. J. Surg. 6, 28-35 (2008)

8. G. Woeste, C. Müller, W.O. Bechstein, et al., World J. Surg. 34, 140 (2010)

9. E. Danse, L. Goncette, A. Kartheuser, JBR-BTR 90, 526 (2007)

10. E. Sacristan, R.A. Baheza, A.O. Rodriguez et al., Proc. 25th IEEE EMBS Conf., 2003, p. 474

11. A. Sato, M. Kataoka, Y. Kuwabara et al., J. Surg. Res. 61, 373 (1996)

12. D.I. Hoult, R.E. Richards, J. Magn. Reson. 24, 71 (1976)

13. D.I. Hoult, P.C. Lauterbur, J. Magn. Reson. 34, 425 (1979)

14. L.L. Arnder, M.D. Shattuck, R.D. Black, Magn. Reson. Med. 35, 727 (1986)

15. T.H. Farmer, G.P. Cofer, G.A. Johnson, Invest. Radiol. 25, $552(1990)$

16. E. Shamonina, V.A. Kalinin, K.H. Ringhofer, L. Solymar, Electron. Lett. 38, 371 (2002)

17. M.C.K. Wiltshire, E. Shamonina, I.R. Young, L. Solymar, Electron. Lett. 39, 215 (2003)

18. A. Radkovskaya, O. Sydoruk, M. Shamonin, et al., Micr. Opt. Tech. Letts. 49, 1054 (2007)

19. L. Solymar, O. Zhuromskyy, O. Sydoruk et al., J. Appl. Phys. 99, 123908 (2006)

20. R.R.A. Syms, T. Floume, I.R. Young et al., Metamaterials 4, $1(2010)$

21. T.G. Hardy, W.G. Pace, J.W. Maney, et al., Dis. Colon Rectum 28, 484 (1985)

22. T.G. Hardy, P.S. Aguilar, W.R.C. Stewart, et al., Dis. Colon Rectum 30, 55 (1987)

23. R. Ravichandran, S. Sundarrajan, J.R. Venugopal, et al., J. Roy. Soc. Interface 7, S559 (2010)

24. C.M. Boutry, H. Chandrahalim, P. Streit, et al., Philos. Trans. Roy. Soc A 370, 2418 (2012)

25. P.M. George, A.W. Lyckman, D.A. LaVan, et al., Biomaterials 26, $3511(2005)$

Cite this article as: H. Kamel, R.R.A. Syms, E.M. Kardoulaki, M. Rea, Surgical wound monitoring by MRI with a metamaterialbased implanted local coil, EPJ Appl. Metamat. 2018, 5, 5 